\title{
Resistance trained athletes using or not using anabolic steroids compared to runners: effects on cardiorespiratory variables, body composition, and plasma lipids
}

\author{
Rachel Yeater, Charles Reed, Irma Ullrich, Anthony Morise, Mark Borsch
}

\begin{abstract}
Objective-To determine whether there is a difference in cardiac size and function as well as in body composition, aerobic capacity, and blood lipids between resistance trained athletes who use anabolic steroids and those who do not, and to compare them to university cross country athletes.

Methods-Four groups of men were evaluated: recreational lifters, $n=11$, lifting $<10 \mathrm{~h} \cdot$ week $^{-1}$; heavy lifters, $n=16$, lifting $>10 \mathrm{~h} \cdot$ week $^{-1}$; steroid users, $n=8$, same as heavy lifters and used steroids; runners, $n=8$, university track members. Echocardiograms, body composition (hydrostatic weighing), maximum oxygen consumption $\left(\mathrm{Vo}_{2}\right)$, and lipids were studied.

Results-As expected, $\mathrm{Vo}_{2}\left(\mathrm{ml} \cdot \mathrm{kg}^{-1} \cdot \mathrm{min}^{-1}\right)$, was greatest in the runners, with no difference among the lifting groups. High density lipoprotein cholesterol in the steroid user group was lower than in heavy lifters or runners. Left ventricular internal diastolic dimension was similar among the groups. The left ventricular mass index of the steroid user group was significantly greater than recreational lifters, at $161 v 103$. There was no difference among heavy lifters (127), runners (124), and steroid users. There was no compromise in diastolic function in any group. There were no differences among groups in resting or exercise blood pressure.
\end{abstract}

Conclusions-Resistance training in the absence of steroid use results in the same positive effects on cardiac dimensions, diastolic function, and blood lipids as aerobic training.

(Br F Sports Med 1996;30:11-14)

Exercise Physiology Program, Department of Medicine,

274 Coliseum,

West Virginia

University,

Morgantown,

West Virginia

26506-6116, USA

R Yeater

C Reed

I Ullrich

A Morise

M Borsch

Correspondence to:

Dr R Yeater.

Accepted for publication

12 October 1995
Key terms: anabolic steroids; cardiac dimensions; blood lipids; resistance training

Although specific numbers are not available, it is widely believed that many athletes involved in sports in which strength is important use anabolic steroids to increase muscle mass. Body building and power lifting are two such sports associated with widespread steroid use. Several adverse effects of steroid use have been identified, including an increase in low density lipoprotein (LDL) cholesterol and a decrease in high density lipoprotein (HDL) cholesterol $^{1-6}$; these adverse lipid changes could result in coronary heart disease if they were allowed to persist for an extensive period. Studies of the effects of resistance training on cardiac muscle size or left ventricular function are conflicting, some showing asymmetric or concentric hypertrophy compared to controls $^{7-10}$ and some showing no significant changes from controls. ${ }^{712}$ Recent studies have compared steroid using resistance trained athletes to non-drug users and have found left ventricular hypertrophy and normal diastolic function in non-steroid using subjects, and concentric hypertrophy with impairment of diastolic function in the drug users. ${ }^{13}{ }^{14}$ In a preliminary study, we have shown an increase in left ventricular mass with normal diastolic function in steroid using body builders and power lifters. ${ }^{15}$

Resistance training without the use of steroids has been shown to be beneficial. We have previously reported ${ }^{16}$ that weight training in the absence of aerobic training may improve maximum oxygen consumption and HDL cholesterol in young men trained for eight weeks. Since resistance training is a popular recreational activity for many, as well as an integral part of the training regimen for most sports, it is important to determine whether resistance training without concomitant steroid use has detrimental effects on cardiac size and function.

The purpose of this study was to determine whether there is a difference in cardiac size and function, body composition, aerobic capacity, and blood lipids between resistance trained athletes who use anabolic steroids and those who do not and compare them to college cross country runners, who represent an aerobically trained group.

\section{Methods}

This cross sectional study examined men who trained at several health clubs in the Morgantown, West Virginia area and members of the West Virginia University cross country team.

A questionnaire was distributed to potential participants to assess length and duration of resistance training and other exercise. In order to preserve confidentiality, no information regarding steroid use was recorded on this form, but those who used steroids were asked 
to put their completed questionnaires in a special envelope.

On the basis of their answers on the questionnaire, resistance trained subjects were assigned to one of three groups. The control group, recreational lifters, consisted of $11 \mathrm{men}$ who were lifters but who trained less than 10 $h$ each week. The heavy lifters group was comprised of 16 men who performed resistance exercise more than $10 \mathrm{~h}$ each week and did not use steroids. The steroid users group was a group of eight men who used various anabolic steroids for at least six months and who engaged in resistance training for at least $10 \mathrm{~h}$ each week. All groups had been doing resistance training for at least two years and performed little or no aerobic activity; their mean age was 21 with a range of 18-30 years.

The eight university cross country athletes were studied during their training season. They were running 50 to 70 miles per week.

Body composition was assessed by hydrostatic weighing. ${ }^{17}$ Girth measurements were made of the neck, chest, biceps, waist, hips, thigh, and calf. Aerobic capacity was measured by a symptom limited maximum treadmill test using the modified Balke protocol for the resistance trained athletes and a Bruce protocol for the runners. ${ }^{18} 19$ Maximum oxygen consumption $\left(\mathrm{V}_{2} \max \right)$ was determined by collecting a $1 \mathrm{~min}$ expired air sample during the last minute of exercise through a low resistance valve into a meteorological balloon. Gas volume was determined with a ParkinsonCowan dry gas meter. Gases were analysed using a Beckman OM-11 oxygen analyser and LB-2 carbon dioxide analyser. Analysers were calibrated with known gases before each measurement. Venous blood was obtained fasting and at least $24 \mathrm{~h}$ after exercise; total and HDL cholesterol and triglycerides were measured enzymatically. LDL cholesterol was calculated by the method of Friedewald. ${ }^{20}$

Cardiac size and function were assessed using Doppler and $M$ mode echocardiography using a General Electric Pass-II phased array sector scanner. The $M$ mode scans were obtained in the long axis parasternal view and were used to determine the thickness of the interventricular septum (IVS), the posterior free wall (PW), and the left ventricular internal diastolic dimension (LVIDD). Free wall thickness was measured at the level of the tips of the

Table 1 Subject characteristics. Values are means (SD)

\begin{tabular}{|c|c|c|c|c|}
\hline & $\begin{array}{l}\text { Regular lifters } \\
(n=11)\end{array}$ & $\begin{array}{l}\text { Heavy lifters } \\
(n=16)\end{array}$ & $\begin{array}{l}\text { Steroid users } \\
(n=8)\end{array}$ & $\begin{array}{l}\text { Runners } \\
(n=8)\end{array}$ \\
\hline Height $(\mathrm{cm})$ & $180(8)$ & $175(4)$ & $177(9)$ & $181(5)$ \\
\hline Weight $(\mathrm{kg})$ & $85(10)^{\mathrm{abc}}$ & $89(15)^{\mathrm{ab}}$ & $97(12)^{a}$ & $69(5)^{c}$ \\
\hline$\%$ Fat & $11(3)^{a}$ & $10(5)^{\mathrm{ab}}$ & $11(5)^{\mathrm{ab}}$ & $5(3)^{b}$ \\
\hline BMI $\left(\mathrm{kg} \cdot \mathrm{m}^{-2}\right)$ & $26(2)^{a}$ & $28(3)^{\mathrm{ac}}$ & $31(3)^{b c}$ & $21(1)^{d}$ \\
\hline $\mathrm{Max} \mathrm{VO}_{2}\left(\mathrm{ml} \cdot \mathrm{kg}^{-1} \cdot \mathrm{min}^{-1}\right)$ & $38(13)^{\mathrm{a}}$ & $43(7)^{\mathrm{a}}$ & $40(4)^{a}$ & $63(6)^{b}$ \\
\hline Resting HR (beats $\cdot \min ^{-1}$ ) & $74(8)$ & $64(10)$ & 79 (11) & $60(10)$ \\
\hline \multirow[t]{2}{*}{ Resting $\mathrm{BP}(\mathrm{mm} \mathrm{Hg})$} & $131(20)$ & $126(14)$ & $130(3)$ & $121(8)$ \\
\hline & $84(11)$ & $81(9)$ & $84(6)$ & $78(9)$ \\
\hline \multirow{2}{*}{ End exercise $\mathrm{BP}(\mathrm{mm} \mathrm{Hg})$} & $194(20)$ & $194(25)$ & $191(17)$ & $180(21)$ \\
\hline & $53(33)$ & $63(30)$ & $77(20)$ & $38(32)$ \\
\hline \multirow{2}{*}{ Recovery BP $(2 \mathrm{~min})(\mathrm{mm} \mathrm{Hg})$} & $172(14)$ & $168(22)$ & $168(20)$ & $165(21)$ \\
\hline & $53(21)^{a}$ & $65(15)^{a}$ & $71(11)^{a}$ & $42(36)^{b}$ \\
\hline
\end{tabular}

$\mathrm{BMI}$, body mass index; HR, heart rate; $\mathrm{BP}$, blood pressure

Groups not sharing the same letter superscripts are significantly different from each other at $P<0.05$. mitral valve leaflets, the other measurements were made at or just below these leaflets.

Left ventricular (LV) mass was calculated using the Penn conventions and formula ${ }^{13}$ :

$$
\begin{aligned}
\mathrm{LV} \text { mass }(\mathrm{g})= & 1.04\left[\mathrm{LVIDD}+\mathrm{IVS}+\mathrm{PW}^{3}-\right. \\
& (\mathrm{LVIDD})^{3}-13.6
\end{aligned}
$$

Left ventricular mass index was expressed as LV mass/body surface area.

Doppler study of the diastolic flow across the mitral valve was taken in the apical four chamber view using a $3.5 \mathrm{mHz}$ phased array transducer.

Flow during early diastole (E) was measured, as well as flow during the atrial contraction (A). Peak velocity was measured as the height of each wave; the velocity integral was calculated as area under each curve. A mean of three cardiac cycles was used for each measurement.

Statistical analysis between groups was assessed by one way analysis of variance. Values are expressed as means(SD).

\section{Results}

Height among the four groups was not different, but the runners were significantly lighter than the heavy lifters and steroid users. The recreational lifter group weighed 85 (10) $\mathrm{kg}$, the heavy lifters 89 (15) kg, the steroid users 97 (12) kg, and the runners 69 (5) kg.

Body mass index was less in the runners than in the other groups; the runners had significantly less fat, 5\%, compared to the other groups: recreational lifters $11 \%$, heavy lifter group $10 \%$, and steroid users $11 \%$ (see table 1 ). The mean circumferences of the neck, biceps, thigh, and chest were greater in the steroid users than the other three groups $(P<0.05)$ (data not shown).

Maximum oxygen consumption was not different among the resistance trained groups, expressed either as $\mathrm{ml} \cdot \mathrm{kg}^{-1} \cdot \mathrm{min}^{-1}$ [recreational lifters 38 (13), lifters group 43 (7), steroid users 40 (4)] or as litres $\mathrm{min}^{-1}$. The runners had the highest $\dot{\mathrm{V}}_{2}$ max of any group [62 (6) $\mathrm{ml} \cdot \mathrm{kg}^{-1} \cdot \mathrm{min}^{-1}, \mathrm{P}<0 \cdot 05$ ]. Resting blood pressure and blood pressure at the end of exercise during the treadmill test were not different among the lifting groups. The diastolic blood pressure during recovery from exercise was lower in the runners $[42(36) \mathrm{mm}$ ] compared to the heavy lifting groups [recreational lifters 53 (21), heavy lifters 65 (15), steroid users 71 (11)]. There was no difference among the heavy lifting groups (see table 1).

Results of plasma lipid determinations are shown in table 2. Total and LDL cholesterol and triglycerides were not different among the groups. The mean HDL cholesterol level of $0.57(0.34) \mathrm{mmol} \cdot \mathrm{litre}^{-1}$ in the steroid group was significantly lower than the values of 1.32 $(0.36)$ and $1.37(0.47)$ in the heavy lifters and the runners respectively. HDL cholesterol was $1.01(0.28) \mathrm{mmol} \cdot$ litre $^{-1}$ in the recreational lifters.

Cardiac dimensions are shown in table 3. Left atrial size was similar among the groups. Although the interventricular septum and posterior wall were larger in the steroid group, 
Table 2 Plasma lipids. Values are means (SD)

\begin{tabular}{|c|c|c|c|c|}
\hline & $\begin{array}{l}\text { Regular lifters } \\
(n=10)\end{array}$ & $\begin{array}{l}\text { Heavy lifters } \\
(n=11)\end{array}$ & $\begin{array}{l}\text { Steroid users } \\
(n=7)\end{array}$ & $\begin{array}{l}\text { Runners } \\
(n=7)\end{array}$ \\
\hline Total cholesterol $\left(\mathrm{mmol} \cdot \text { litre }^{-1}\right)^{\star}$ & $4 \cdot 14(1 \cdot 24)$ & $3.94(0.41)$ & $4 \cdot 48(1 \cdot 27)$ & $4.04(0.65)$ \\
\hline HDL-C (mmol-litre $\left.{ }^{-1}\right)$ & $1.01(0.28)^{2 b}$ & $1.32(0.36)^{2}$ & $0.57(0.34)^{\mathrm{ab}}$ & $1.37(0.47)^{2}$ \\
\hline LDL-C $\left(\mathrm{mmol}^{-}\right.$litre $\left.^{-1}\right)$ & $2 \cdot 72(1.01)$ & $2 \cdot 31(0 \cdot 39)$ & $3.37(1.45)$ & $2.31(0.52)$ \\
\hline Triglycerides $\left(\mathrm{mmol} \cdot\right.$ litre $\left.^{-1}\right) \dagger$ & $0.94(0.29)$ & $0.92(0.43)$ & $1 \cdot 19(0.35)$ & $0.86(0.25)$ \\
\hline
\end{tabular}

${ }^{\star} \mathrm{mmol} \cdot \mathrm{litre}^{-1} \div 0 \cdot 0259=\mathrm{mg} \cdot \mathrm{dl}^{-1} ;$ tmmol $\cdot$ litre $^{-1} \div 0.0113=\mathrm{mg} \cdot \mathrm{dl}^{-1}$

HDL-C, high density lipoprotein cholesterol; LDL-C, low density lipoprotein cholesterol.

Groups not sharing the same superscript are significantly different from each other at $P<0.05$.

Table 3 Cardiac dimensions. Values are means (SD)

\begin{tabular}{|c|c|c|c|c|}
\hline & $\begin{array}{l}\text { Regular lifters } \\
(n=11)\end{array}$ & $\begin{array}{l}\text { Heavy lifters } \\
(n=16)\end{array}$ & $\begin{array}{l}\text { Steroid users } \\
(n=8)\end{array}$ & $\begin{array}{l}\text { Runners } \\
(n=8)\end{array}$ \\
\hline $\mathrm{LA}(\mathrm{mm})$ & $37(3 \cdot 8)$ & $37 \cdot 0(5 \cdot 2)$ & $37 \cdot 8(1 \cdot 8)$ & $34 \cdot 6(4 \cdot 4)$ \\
\hline IVS (mm) & $9 \cdot 3(1 \cdot 3)$ & $9 \cdot 5(2 \cdot 4)$ & $12 \cdot 4(3 \cdot 4)$ & $9 \cdot 8(2 \cdot 7)$ \\
\hline PW (mm) & $9.9(1.7)$ & $11 \cdot 6(2 \cdot 4)$ & $13 \cdot 1(3 \cdot 4)$ & $11 \cdot 1(3 \cdot 1)$ \\
\hline IVS/PW & $0.95(0.17)$ & $0.84(0 \cdot 20)$ & $0.94(0.10)$ & $0.90(0 \cdot 25)$ \\
\hline LVIDD (mm) & $51 \cdot 7(8 \cdot 1)$ & $53.9(4 \cdot 3)$ & $54.0(6.9)$ & $51 \cdot 1(5 \cdot 3)$ \\
\hline LVMASS (g) & $212(39)^{2 c}$ & $268(94)^{\text {abc }}$ & $345(89)^{\mathrm{bc}}$ & $237(76)^{\mathrm{ac}}$ \\
\hline LVMASS I $\left(\mathrm{g} \cdot \mathrm{m}^{-2}\right)$ & $103(16)^{a}$ & $127(43)^{2 b}$ & $161(36)^{b}$ & $124(35)^{2 b}$ \\
\hline
\end{tabular}

LA, left atrium; IVS, interventricular septum; PW, left ventricular posterior wall; LVIDD, left ventricular internal diastolic diameter; LVMASS, left ventricular mass; LVMASS I, left ventricular mass $\div$ body surface area.

Groups not sharing the same superscripts are significantly different from each other at $\mathrm{P}<0.05$.

this was not statistically significant. There was no disproportionate increase in septal size compared to the posterior wall in any of the lifting groups. The left ventricular internal diastolic dimension was similar among the groups.

The mass of the left ventricle was $212 \mathrm{~g}$ in recreational lifters, $268 \mathrm{~g}$ in heavy lifters, 345 $\mathrm{g}$ in steroid users, and $237 \mathrm{~g}$ in runners The value for the steroid users was significantly greater than the recreational lifter group $(P<0.05)$. Since heart size increases with body size, measurement may be normalised by dividing heart size by body surface area, resulting in a mass index. The left ventricular mass index was 161 in steroid users, 127 in heavy lifters, 103 in recreational lifters, and 124 in runners. The left ventricular mass index of steroid users was significantly greater than in the recreational lifters $(P<0.05)$; there was no difference between heavy lifters and runners.

The ratio of early $(\mathrm{E})$ to late $(\mathrm{A})$ diastolic filling was not significantly different among the groups. Peak E and peak $A$, normalised for mitral valve size, were significantly higher $(P<0.05)$ in steroid users than in runners; the values for heavy lifter and runners were not significantly different. Ejection fraction and cardiac output were similar among the groups.

\section{Discussion}

The results of this cross sectional study indicate that athletes using steroids do have increased skeletal muscle mass, as evidenced by anthropometric measurements and body composition. Although there was no difference in the amount of time the heavy lifter and steroid user groups engaged in training, it is possible that the aggressive feelings associated with steroid use enabled the steroid using subjects to lift heavier weights with more intensity during their exercise sessions.

Our subjects admitted using a variety of anabolic steroids with varying regimens, given both orally and by injection. Since the steroids were purchased illegally and were unavailable for inspection, it is not possible to know the exact composition or the actual amounts the subjects received. We did not assess plasma or urine drug levels; however, we believe these subjects' histories accurately reflect steroid usage as do their low plasma HDL cholesterol levels. Conversely, members of the other groups were adamant in stating they did not use these compounds, and their plasma lipid levels support their statements.

Aerobic capacity, assessed by treadmill testing was, as expected, highest in the cross country athletes. Although the cross country athletes may have had a slight advantage during testing since they were assessed with a protocol that involved running, and therefore possibly greater muscle involvement than the walking protocol of the lifters, we felt the difference was unavoidable. Since the lifters were unaccustomed to running, it was felt their legs might fatigue before their cardiovascular system if a running protocol on a steep incline were used. Conversely, cross country athletes would be at such an extreme elevation in a walking protocol that they might have difficulty with leg cramps if such a protocol was used to assess their $\dot{\mathrm{VO}}_{2} \max$. Therefore we felt we used protocols that would elicit the optimal aerobic performance from each group.

The resistance trained athletes had significantly lower $\mathrm{VO}_{2}$ max than the cross country athletes, although they were not different from each other. All of the resistance trained athletes showed at least average or above average (Palo Alto norms) $\mathrm{VO}_{2}$ max for their age group, even though they participated in no aerobic training. We have previously shown that weight training without aerobic conditioning can increase $\mathrm{VO}_{2}$ max in healthy young men. ${ }^{16}$

Blood pressure values at rest, during exercise, and during recovery were normal for all groups. This was surprising since it is widely believed that blood pressure at rest and during exercise is increased as a result of anabolic steroid use. ${ }^{22}$ It might be that blood pressure response to anabolic steroids is dose related, and although the subjects appeared to have had a significant dose of steroids to affect both skeletal and cardiac muscle mass, the dose may not have been high enough to affect blood pressure.

The adverse effects of steroids on plasma lipids (increased LDL and decreased HDL cholesterol) have been reported previously ${ }^{1-6}$ and may portend increased potential for development of atherosclerotic cardiovascular disease. Cardiac muscle hypertrophy associated with limited vascular supply may result in more disease than would be noted without hypertrophy.

Left ventricular mass was significantly increased in the steroid users, but the steroid users were also heavier, with larger anthropometric measurements. Even after correction for larger body size by using body surface area (index), the steroid users still had a significantly larger index than the recreational lifters. They were not larger than the heavy lifters or runners probably because of the greater standard deviation in these groups. 
In a recent echocardiographic study of 947 elite Italian athletes, ${ }^{22}$ a left ventricular wall thickness greater than $13 \mathrm{~mm}$ was found to be very uncommon, and these data suggested that the upper limit of left ventricular wall thickness that could be induced by athletic training without causing enlargement of the end diastolic cavity was $16 \mathrm{~mm}$. In our study, $25 \%$ of the steroid group had a left ventricular wall thickness more than $13 \mathrm{~mm}$, with one subject having an interventricular septum thickness of $19 \mathrm{~mm}$. The Italian athletes had a posterior wall (PW) thickness range from 7 to $13 \mathrm{~mm}$. In our study, $50 \%$ of the steroid group had a PW thickness greater than $13 \mathrm{~mm}$, with one athlete having a value of $20 \mathrm{~mm}$. Only $25 \%$ (four of 16) of the heavy lifters had a PW over $13 \mathrm{~mm}$, while in only one runner was the value over 13. None of the recreational lifters had a PW greater than $13 \mathrm{~mm}$. Despite this increase in muscle mass, there was no compromise of diastolic function in the steroid users. This is in conflict with a recent study of Italian steroid using body builders who had left ventricular hypertrophy and decreased diastolic function compared to non-steroid-using body builders and sedentary controls. ${ }^{14}$ Our study supported the findings of Thompson et $a l^{23}$ with regard to lack of compromise of diastolic function, but did not support their finding that there was no increase in cardiac mass in steroid users compared to non-drug-using weight lifters. Our data indicate that anabolic steroid use concomitant with resistance training for more than than 10 hours per week results in significant cardiac hypertrophy with no compromise in diastolic function. The conflicting results that continue to be found in research studies may be due to the different types and varying doses of steroids being taken by the athletes. It may be that anabolic steroids stimulate cardiac muscle hypertrophy in a manner similar to their effect on skeletal muscle. These effects may be further amplified by other stimuli such as exercise.

It has been widely believed that the response of the heart to resistance training compared to aerobic training is different. Resistance exercise, such as weight lifting, is thought to result in increased cardiac muscle mass at the expense of ventricular cavity size, the result of a pressure load..$^{8-11}$ Aerobic training, on the other hand, is thought to result primarily in increased left ventricular cavity size with no change in wall thickness, the result of a volume load. ${ }^{911}$ Our results, like those of Roy et al, ${ }^{12}$ indicate that resistance training in the absence of steroid use is similar to aerobic training with respect to its effects on cardiac dimensions and functions.

\section{CONCLUSIONS}

Resistance training in the absence of steroid use results in the same positive effects on cardiac dimensions and diastolic function as aerobic training. Resistance training in the absence of steroid use does not result in adverse effects on resting or exercise blood pressure or on blood lipids.

1 Zuliani Y, Bernardini B, Catapano A, Campana $M$ Cerioli G, Spattini M. Effects of anabolic steroids, testosterone, and HGH on blood lipids and echotestosterone, and HGH on blood lipids and echocardiographic param

2 Graham M, Ballantyne D. Lipoprotein analysis in body builders. Int $\mathcal{f}$ Cardiol 1987;1 7:281-6.

3 Hurley B, Seals D, Hagberg J, Goldberg A, Ostrove S, Holloszy J, et al. High-density-lipoprotein cholesterol in body builders v power lifters. $\mathscr{F} A M A$ 1984;252:507-13.

4 Frohlich J, Kullmer T, Urhausen A, Bergmann $R$ Kinderman W. Lipid profile of body builders with and without self-administration of anabolic steroids. $\mathcal{F} \mathrm{Appl}$ Physiol 1989;59:98-103.

5 Alen M, Rahkida P. Anabolic-androgenic steroid effects on endocrinology and lipid metabolism in athletes. Sports Med 1988;6:327-32

6 Baldo-Enzi G, Giada F, Zuliani G, Baroni L, Vitale E Enzi G, et al. Lipid and apoprotein modifications in body buil $\mathrm{G}$, et al. Lipid and apoprotein modifications in body builders during and after self-adminis
steroids. Metabolism 1990;39:203-8.

7 Urhausen A, Holpes R, Kinderman W. One- and twodimensional echocardiography in body builders using anabolic steroids. Eur $\mathcal{F}$ Appl Physiol 1989;58:633-40.

8 Fleck SJ, Henke C, Wilson W. Cardiac MRI of elite junio Olympic weight lifters. Int $\mathcal{F}$ Sports Med 1989;10:329-33.

9 Longhurst JC, Kelly AR, Gonyea WJ, Mitchell JH. Echocardiographic left ventricular masses in distance runners and weight lifters. F Appl Physiol 1980 (Resp Environ Exerc Physiol);48:154-162.

10 Menapace F, Hammer WJ, Ritzer TF, Kessler KM Warner HF, Spann JF, et al. Left ventricular size in competitive weight lifters: an echocardiographic study. Med Sci Sports Exerc 1982;14:72-5.

11 Morganroth J, Maran BJ, Henry WL, Epstein SE. Comparative left ventricular dimensions in trained athletes. parative left ventricular dim

12 Roy A, Doyon M, Dumesnil JJ, Landry F. Endurance vs. Strength training: comparison of cardiac structures using normal predicted values. F Appl Physiol 1988;64:2552-7.

13 Pearson A, Schiff M, Mrosek D, Labovitz A, Williams G Left ventricular diastolic function in weight lifters. $A m \mathcal{F}$ Cardiol 1986;58:1254-9.

14 Piccoli B, Giada F, Benettin A, Sartori F, Piccolo E Anabolic steroid use in body builders: An echocardiographic study of left ventricle morphology and function. Int $\mathcal{F}$ Sports Med 1991;12:408-12.

15 Ullrich I, Younis M, Yeater R, Borsch M, Zanke C. Cardiac hypertrophy in weight lifters: only in steroid users? Med Sci Sports Exerc 1990;22:S64.

16 Ullrich I, Reid C, Yeater R. Increased HDL cholesterol levels with a weight lifting program. South Med $\mathcal{f}$ 1987;80:328-31.

17 Warner J, Yeater R, Sherwood L, Weber K. A hydrostatic weighing method using total lung capacity and a small tank. Br F Sports Med 1986;20:17-21.

18 Balke B, Ware R. An experimental study of physical fitness of Air Force personnel. US Armed Forces Med $\mathcal{F} 1959$ 10:675-8.

19 Bruce R. Exercise testing for ventricular function. $N$ Engl f Med 1977;296:671.

20 Friedewald W, Levy R, Fredrickson D. Estimation of the concentration of low-density lipoprotein cholesterol in plasma without the use of the preparative ultracentrifuge. Clin Chem 1972;18:499-502.

21 Riebe D, Fernhall B. Anabolic steroid use and blood pressure response to dynamic exercise. Med Sci Sport Exerc 1991;23:S58.

22 Pellicia A, Maron B, Spataro A, Proschan M, Spirito P. The upper limit of physiologic cardiac hypertrophy in highly

23 Thompson P, Sadoniantz A, Cullinane E, Bodziang K, Catlin D, Torek-Boten G, et al. Left ventricular function is not impared in weight lifters who use anabolic steroids. f Am Coll Cardiol 1992;19:278-82. 